\title{
AN EXERCISE ON FIBONACCI REPRESENTATIONS
}

\author{
JEAN BERSTEL ${ }^{1}$
}

\begin{abstract}
We give a partial answer to a question of Carlitz asking for a closed formula for the number of distinct representations of an integer in the Fibonacci base.
\end{abstract}

Mathematics Subject Classification. 68R15, 68R05.

\section{INTRODUCTION}

In 1968, Carlitz [2] derived formulas for the number of distinct representations of an integer $n$ in the Fibonacci number system. He wondered whether a closed formula exists for this number, as a function of $n$. The aim of this note is to give a partial answer to this question. The answer is only partial because our formula is not really "closed" in the sense that it implies evaluation of a product of matrices.

It is well-known that integers may have more than one representations when expressed in the Fibonacci number system. This is due to the fact that two adjacent 1 's in a representation may be replaced by two 0's with a carry to the next position. More precisely, the number represented by a sequence of 0 's and 1's is invariant under the congruence generated by $011 \equiv 100$ (precise notation will be given later). It was discovered by Schützenberger (personal communication) that iteration of the replacement of 011 by 100 is a rational transduction. As a consequence, the normalized representation(i.e. the representation without factor 11) can be computed by a finite transducer. Subsequently, the transducer has been greatly improved by Sakarovitch (for the history, see Frougny and Sakarovitch [5]), and we now have a letter to letter unambiguous transducer that computes this rational relation. By reverting the transducer, we get an automaton that computes the set of all representations of an integer in the Fibonacci number system. Since the transducer is unambiguous, counting the size of the output set for a given word yields the desired expression. This value can be computed as usual by evaluating a product of matrices.

\footnotetext{
1 Institut Gaspard Monge (IGM), Université de Marne-la-Vallée, 5 boulevard Descartes, 77454 Marne-la-Vallée Cedex 2, France; e-mail: Jean.Berstel@univ-mlv.fr
} 
As we shall see, the transducer has four states (this seems to be minimal) and therefore the matrices are $4 \times 4$ matrices. However, a closer look on the values leads to another matrix expression where the matrices are only 2 by 2 . The coefficients of these matrices admit rather simple expressions. They appear to be obtained by a kind of summation.

It remains to determine whether this new expression is limited to the Fibonacci representation, or whether it is just a simple case of a more general situation. In the last section, we will show that the latter situation holds. Similar expressions can be obtained for all Ostrowski number systems. These number systems play an analogue role, compared to the Fibonacci system, as Sturmian sequences compared to the infinite Fibonacci word.

\section{Notation}

Fibonacci numbers are defined by $F_{0}=1, F_{1}=2, F_{n+2}=F_{n+1}+F_{n}$ for $n \geq 0$. Every positive integer $n$ admits a representation

$$
n=F_{m_{1}}+F_{m_{2}}+\cdots+F_{m_{r}}
$$

with $m_{1}>m_{2}>\cdots>m_{r} \geq 0$. It is convenient to write this representation as a word over the alphabet $\{0,1\}$, putting a 1 whenever the corresponding number is in the sum (1) and a 0 otherwise. Thus, for instance $32=21+5+3+2+1$, and the corresponding word is 1001111. A result by Zeckendorff [10] states that the representation (1) is unique provided $m_{i} \geq 2+m_{i+1}$, that is if there are no consecutive 1 's in the associated word. Such a representation (that we call the Zeckendorff representation) always exists. A simple way to transform a given representation into the Zeckendorff representation is to repeatedly replace a factor 011 by 100 (possibly adding a leading 0 if necessary). The Zeckendorff representation of 32 is 1010100. Two representations are equivalent if they represent the same number.

The aim of this note is to compute the number $R(n)$ of equivalent representations of $n$ in the Fibonacci number system. For instance, $R(32)=4$, because 32 has the four representations 1010100, 1010011, 1001111, and 111111. We compute in fact this number as a function $R(\langle n\rangle)$, where $\langle n\rangle$ denotes the Zeckendorff representation of $n$. Some experimentation shows that the behavior of $R$ is rather erratic. For instance, all numbers $F_{m}-1$ only have one representation, namely $101010 \cdots 101$. In other cases, there are linearly many representations. Consider the word $(100)^{m}$. Each of the $m$ blocs 100 may or may not be replaced by 011 . Thus, there are $2^{m}$ representations of a number $n$ whose size is approximately $\tau^{3 m}$ where $\tau=\frac{1}{2}(1+\sqrt{5})$ is the golden ratio.

\section{A TRANSDUCER}

A way to compute all representations from the Zeckendorff representation (or inversely to compute the Zeckendorff representation from any representation) is 
to repeatedly apply the rewriting rule $100 \rightarrow 011$ (or its converse). Although one application of a rewriting rule is a rational transduction, iteration of a rational transduction usually does not lead to a rational transduction (for recent results on iteration of rational transductions, see Simplot and Terlutte [8,9]). It happens that in the case of the special rewriting rule $100 \rightarrow 011$, iteration is still a rational transduction. The transducer given in figure 1 is taken from the forthcoming book of Sakarovitch [7]. This transducer computes, for any word starting with a 0 , a word of the same length which is the Zeckendorff representation of the integer represented by the input, perhaps with some leading 0's to make the output of the same length. For instance, inputting 01001111 yields as output 01010100. Recall a quite remarkable but general result saying that a length-preserving rational transduction can always be realized by a letter-to-letter transducer (see e.g. Eilenberg [3] or Sakarovitch [7]). The transduction realized by our transducer is a function, and moreover the transducer is unambiguous, that is for each pair $p, q$ of states, and each pair $(x, y)$ of words, there is at most one path from $p$ to $q$ labeled by $(x, y)$.

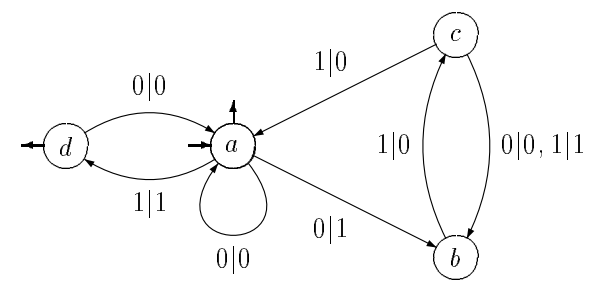

FIGURE 1. Fibonacci normalizer.

Consider the reversed transducer obtained by exchanging input and output labels. The transduction realized by this transducer takes as inputs only Zeckendorff representations and outputs, for each input word $w$, the set of all equivalent representations of the same length (with leading 0's). Since the transducer is unambiguous the number of equivalent representations is the number of successful paths with input label $w$. Thus, we may forget outputs. We consider only the automaton given in Figure 2 and ask for the number of successful paths for a given word. Computation of the number of successful paths for a given word is routine.

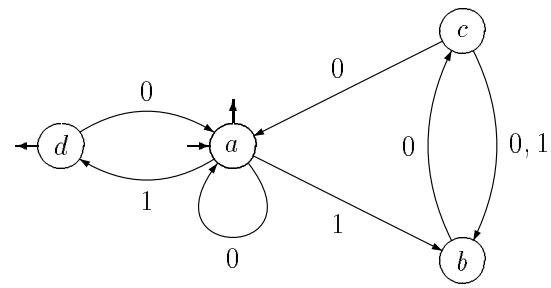

Figure 2. Fibonacci automaton. 
For each letter $a$, we consider a matrix $\mu(a)$, where each coefficient $\mu(a)_{p, q}$ equals the number of edges from state $p$ to state $q$ labeled with $a$. Since the automaton is unambiguous, these coefficients are always 0 or 1 . Extending this matrix representation to words by product, $\mu(w)_{p, q}$ is the number of paths labeled $w$ leading from $p$ to $q$. Thus the number of successful paths for $w$ is the number $\lambda \mu(w) \gamma$, where the row vector $\lambda$ (the column vector $\gamma$ ) is defined by $\lambda_{p}=1\left(\gamma_{p}=1\right)$ if $p$ is an initial (terminal) state, and $=0$ otherwise. Here, $\lambda=\left(\begin{array}{llll}1 & 0 & 0 & 0\end{array}\right)$ and

$$
\mu(0)=\left(\begin{array}{llll}
1 & 0 & 0 & 0 \\
0 & 0 & 1 & 0 \\
1 & 1 & 0 & 0 \\
1 & 0 & 0 & 0
\end{array}\right), \quad \mu(1)=\left(\begin{array}{llll}
0 & 1 & 0 & 1 \\
0 & 0 & 0 & 0 \\
0 & 1 & 0 & 0 \\
0 & 0 & 0 & 0
\end{array}\right), \quad \gamma=\left(\begin{array}{l}
1 \\
0 \\
0 \\
1
\end{array}\right)
$$

Proposition 2.1. Let $w$ be the Zeckendorff representation of $n$. The number of representations of $n$ in the Fibonacci number system is $R(n)=\lambda \mu(w) \gamma$.

$$
\text { For instance, } \mu(1010100)=\left(\begin{array}{cccc}
4 & 3 & 0 & 0 \\
0 & 1 & 0 & 0 \\
1 & 1 & 0 & 0 \\
0 & 1 & 0 & 0
\end{array}\right) \text { and consequently } R(32)=4 \text {. }
$$

\section{A Shorter EXPRESSION}

For any $d \geq 0$, define a matrix $M(d)$ by

$$
M(d)=\left(\begin{array}{cc}
1 & 1 \\
\lfloor d / 2\rfloor & \lceil d / 2\rceil
\end{array}\right)
$$

For instance,

$$
M(0)=\left(\begin{array}{ll}
1 & 1 \\
0 & 0
\end{array}\right), M(1)=\left(\begin{array}{ll}
1 & 1 \\
0 & 1
\end{array}\right), M(2)=\left(\begin{array}{ll}
1 & 1 \\
1 & 1
\end{array}\right), M(3)=\left(\begin{array}{ll}
1 & 1 \\
1 & 2
\end{array}\right)
$$

If the integer $n$ is written as

$$
n=F_{m_{1}}+F_{m_{2}}+\cdots+F_{m_{r}}
$$

with $m_{i} \geq 2+m_{i+1}$, set

$$
d_{i}=m_{i}-m_{i+1}-1,(i=1, \ldots, r-1), \quad d_{r}=m_{r} .
$$

Then the word $\langle n\rangle$ has the expression

$$
\langle n\rangle=10^{d_{1}} 10^{d_{2}} \cdots 10^{d_{r}} .
$$


Proposition 3.1. Let $\langle n\rangle=10^{d_{1}} 10^{d_{2}} \cdots 10^{d_{r}}$ be the Zeckendorff representation of a positive integer $n$. The number of representations of $n$ in the Fibonacci number system is

$$
R(n)=\left(\begin{array}{ll}
1 & 1
\end{array}\right) M\left(d_{1}\right) M\left(d_{2}\right) \cdots M\left(d_{r}\right)\left(\begin{array}{l}
1 \\
0
\end{array}\right) .
$$

For $r=1$, this is Theorem 2 of Carlitz [2]. In this case, $n=F_{d}$ for some $d$ and $\left\langle F_{d}\right\rangle=10^{d}$. Consequently, $R\left(F_{d}\right)=1+\lfloor d / 2\rfloor$. As another example, consider again $n=32=21+8+3$. Then $\langle n\rangle=1010100$, whence $\left(d_{1}, d_{2}, d_{3}\right)=(1,1,2)$ and

$$
R(32)=\left(\begin{array}{ll}
1 & 1
\end{array}\right)\left(\begin{array}{ll}
1 & 1 \\
0 & 1
\end{array}\right)\left(\begin{array}{ll}
1 & 1 \\
0 & 1
\end{array}\right)\left(\begin{array}{ll}
1 & 1 \\
1 & 1
\end{array}\right)\left(\begin{array}{l}
1 \\
0
\end{array}\right)=4 .
$$

Observe that the formula of the expression is close to that derived from the automaton, where the matrices $M(d)$ are "condensed" by considering each block $10^{d}$ as a "letter".

Proof. By induction on the number $r$ in the expression $\langle n\rangle=10^{d_{1}} 10^{d_{2}} \cdots 10^{d_{r}}$. The lengths of two representations of a positive integer $n$ differ by at most one. Denote by $G(n)$ the number of long representations, and by $P(n)$ the number of short representations, so that $R(n)=G(n)+P(n)$. Consider the Zeckendorff representation of $\langle n\rangle=10^{d} 10^{d_{2}} \ldots 10^{d_{r}}$ and let $m$ be the integer with representation $10^{d_{2}} \cdots 10^{d_{r}}$. Then $G(n)=R(m)=G(m)+P(m)$, because a long representation of $n$ can only be obtained by keeping unchanged the leading block $10^{d}$. Concerning short representations, there are two cases. If $d$ is even, then there are $d / 2$ short representations of $10^{d}$, and each short representation of $m$ contributes a suffix of a short representation of $n$ when completed with one leading 0 . Thus $P(n)=d / 2 R(m)$.

If $d$ is odd, there is one special representation of $10^{d}$, namely the representation ending in 10. This last block 10, together with any short representation of $m$ contributes to two representations of $n$, the first having a joining block 100 and the second 011. It follows that $P(n)=\lfloor d / 2\rfloor R(m)+P(m)$. Grouping these formulas, and using the equality $R(m)=G(m)+P(m)$, we get the relation

$$
\left(\begin{array}{l}
G(n) \\
P(n)
\end{array}\right)=\left(\begin{array}{cc}
1 & 1 \\
\lfloor d / 2\rfloor & \lceil d / 2\rceil
\end{array}\right)\left(\begin{array}{l}
G(m) \\
P(m)
\end{array}\right)
$$

The result follows.

Let us mention that the argument of the proof also holds when the integers $d_{i}$ are zero. This means that we do not need to start with the Zeckendorff representation.

Corollary 3.2. Let $n$ be a positive integer, and let $w=10^{d_{1}} 10^{d_{2}} \cdots 10^{d_{r}}$ be any representation of $n$ in the Fibonacci number system. The number $R(n, w)$ of representations of $n$ which are lexicographically smaller than or equal to $w$ is given by

$$
R(n, w)=\left(\begin{array}{ll}
1 & 1
\end{array}\right) M\left(d_{1}\right) M\left(d_{2}\right) \cdots M\left(d_{r}\right)\left(\begin{array}{l}
1 \\
0
\end{array}\right) .
$$




\section{Ostrowski nUmber System}

As pointed out by Brown [1], Zeckendorff representations exist in a much larger framework, and goes back at least to Ostrowski [6]. This and related number systems were studied in particular by Fraenkel [4] (see also the references there and in [1]). It is convenient, for the exposition given here, to modify slightly the numbering found in the literature.

Consider a sequence $a=\left(a_{0}, a_{1}, \ldots, a_{h}, \ldots\right)$ of integers with $a_{0} \geq 0, a_{h} \geq 1$ for $h \geq 1$. Define a sequence $\left(q_{h}\right)$ by $q_{-1}=q_{0}=1$ and $q_{h+1}=a_{h} q_{h}+q_{h-1}$ for $h \geq 0$. Every positive integer $n$ admits a representation

$$
n=y_{t} q_{t}+\cdots+y_{0} q_{0}
$$

with $y_{t}>0$ and $0 \leq y_{h} \leq a_{h}$ for $0 \leq h \leq t$. The representation is unique provided $y_{h}=a_{h}$ implies $y_{h-1}=0$ for $h>0$. A number system of this kind is called an Ostrowski system, and the unique representation is the Ostrowski representation. Of course, taking $a_{h}=1$ for all $h$, the numbers $q_{h}$ are the Fibonacci numbers, and the condition for uniqueness, namely that $y_{h}=a_{h}$ implies $y_{h-1}=0$, precisely means that there are no adjacent 1's in the representation. Thus the Fibonacci number system is the simplest Ostrowski system. As another example, consider the sequence $a$ starting with $(2,2,3,2,2,2,3)$. A straightforward computation gives $q_{1}=3, q_{2}=7, q_{3}=24, q_{4}=55, q_{5}=134, q_{6}=323$. It follows that the integer $n=660$ has the following six representations in this system:

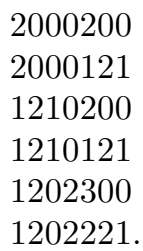

The first is the Zeckendorff representation. If we try to derive formulas similar to those given for the Fibonacci number system, it seems that the automatatheoretic approach will be difficult to extend because the sequence $a$ needs not to have bounded coefficients, and therefore there is no finite "alphabet". Moreover, the position of an integer in the sequence $a$ is quite relevant for the computation, even in the case of bounded coefficients. This cannot be memorized by a finite automaton, unless the sequence $a$ is periodic.

It appears that the short formula of the previous section admits a generalization to the case of Ostrowski number systems. To do this, we consider an integer $n$ written in such a system and, as for the Fibonacci system, we group together consecutive 0 's. Then the representation writes as

$$
b_{1} 10^{d_{1}} b_{2} 10^{d_{2}} \cdots b_{r} 10^{d_{r}}
$$


where $b_{1}, \ldots b_{r}>0$. An integer $b_{h}$ is maximal if it takes the maximal value that is allowed in that position. As an example, in the representation $2000200=20^{3} 20^{2}$, one has $b_{1}=b_{2}=2$. Since the digits in position 2 and 6 can grow up to 3 (because $\left.a_{2}=a_{6}=3\right)$, neither $b_{1}$ nor $b_{2}$ is maximal, despite the fact that the representation is the Zeckendorff representation. In the Fibonacci system, each $b_{i}$ is maximal, because every nonzero digit must be 1 .

In order to state the expression for the number of representations, we introduce matrices $M^{\prime}(d)$ by

For instance

$$
M^{\prime}(d)=\left(\begin{array}{cc}
0 & 0 \\
1+\lfloor d / 2\rfloor & 1+\lceil d / 2\rceil
\end{array}\right)
$$

$$
M^{\prime}(0)=\left(\begin{array}{ll}
0 & 0 \\
1 & 1
\end{array}\right), M^{\prime}(1)=\left(\begin{array}{ll}
0 & 0 \\
1 & 2
\end{array}\right), M^{\prime}(2)=\left(\begin{array}{ll}
0 & 0 \\
2 & 2
\end{array}\right), M^{\prime}(3)=\left(\begin{array}{ll}
0 & 0 \\
2 & 3
\end{array}\right) .
$$

Proposition 4.1. Let $\langle n\rangle=b_{1} 10^{d_{1}} b_{2} 10^{d_{2}} \cdots b_{r} 10^{d_{r}}$ be the Zeckendorff representation of a positive integer $n$ in some Ostrowski number system. The number of representations of $n$ in this system is

$$
R(n)=\left(\begin{array}{ll}
1 & 1
\end{array}\right) N\left(d_{1}\right) N\left(d_{2}\right) \cdots N\left(d_{r}\right)\left(\begin{array}{l}
1 \\
0
\end{array}\right)
$$

where $N\left(d_{h}\right)=M\left(d_{h}\right)$ if $b_{h}$ is maximal, and $N\left(d_{h}\right)=M^{\prime}\left(d_{h}\right)$ otherwise.

As an example, according to the proposition, the number of representations of 660 in our running system is

$$
\left(\begin{array}{ll}
1 & 1
\end{array}\right)\left(\begin{array}{ll}
0 & 0 \\
2 & 3
\end{array}\right)\left(\begin{array}{ll}
0 & 0 \\
2 & 2
\end{array}\right)\left(\begin{array}{l}
1 \\
0
\end{array}\right)=6
$$

Proof. The argument of the proof is quite similar to that of Proposition 3.1. Long and short representations have to be replaced by maximal and sub-maximal representations. A representation of $n$ is maximal if its leading nonzero digit (that we denoted $b_{1}$ ) is maximal. As we have seen, a Zeckendorff representation needs not to be a maximal representation.

Denote by $H(n)$ the number of maximal representations, and by $S(n)$ the number of sub-maximal representations, so that $R(n)=H(n)+S(n)$. Consider the Zeckendorff representation $\langle n\rangle=b_{1} 10^{d_{1}} b_{2} 10^{d_{2}} \cdots b_{r} 10^{d_{r}}$ and let $m$ be the integer represented by $b_{2} 10^{d_{2}} \cdots b_{r} 10^{d_{r}}$. If $b_{1}$ is maximal, then $H(n)=R(m)$ because a maximal representation of $n$ can only be obtained by keeping unchanged the leading block $b_{1} 10^{d_{1}}$. If $b_{1}$ is not maximal, then $H(n)=0$.

Concerning sub-maximal representations, the argument of the previous proof transposes. Observe first that there are $1+\lfloor d / 2\rfloor$ representations of $b_{1} 0^{d}$, and that they are contribute sub-maximal representations of $n$ because $b_{1}$ is not maximal. We consider two cases. If $d=d_{1}$ is even, then $S(n)=(1+d / 2) R(m)$. If $d$ is odd, then there is one special representation of the leading block $b_{1} 0^{d}$, namely 
the representation ending with a $c 0$ for some digit $c>0$. This block, together with the initial letter $b$ of any sub-maximal representation of $m$, contributes to two representations of $n$, the first containing the block $c 0 b$ and the second having the block $(c-1) a_{i}(b+1)$ for a convenient index $i$. It follows that $S(n)=(1+$ $\lfloor d / 2\rfloor) R(m)+S(m)$. Grouping these formulas, one gets the relation

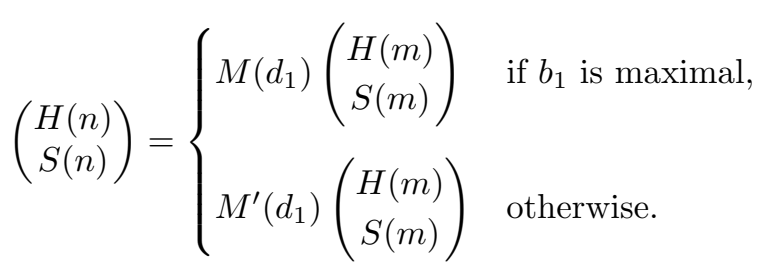

The result follows.

\section{A FINAL REMARK}

As already mentioned, the behavior of $R(n)$ is rather irregular as a function of $n$. Perhaps, some better description could be obtained for the average value of $R(n)$, as it happens to be the case for other number-theoretic functions.

\section{REFERENCES}

[1] T.C. Brown, Descriptions of the characteristic sequence of an irrational. Canad. Math. Bull. 36 (1993) $15-21$.

[2] L. Carlitz, Fibonacci representations. Fibonacci Quarterly 6 (1968) 193-220.

[3] S. Eilenberg, Automata, Languages, and Machines, Vol. A. Academic Press (1974).

[4] A.S. Fraenkel, Systems of numeration. Amer. Math. Monthly 92 (1985) 105-114.

[5] C. Frougny and J. Sakarovitch, Automatic conversion from Fibonacci representation to representation in base $\varphi$ and a generalization. Int. J. Algebra Comput. 9 (1999) 51-384

[6] A. Ostrowski, Bemerkungen zur Theorie der Diophantischen Approximation I. Abh. Math. Sem. Hamburg 1 (1922) 77-98.

[7] J. Sakarovitch, Éléments de théorie des automates. Vuibert (to appear).

[8] D. Simplot and A. Terlutte, Closure under union and composition of iterated rational transductions. RAIRO: Theoret. Informatics Appl. 34 (2000) 183-212.

[9] D. Simplot and A. Terlutte, Iteration of rational transductions. RAIRO: Theoret. Informatics Appl. 34 (2000) 99-129.

[10] E. Zeckendorff, Représentation des nombres naturels par une somme de nombres de Fibonacci ou de nombres de Lucas. Bull. Soc. Royale Sci. Liège 42 (1972) 179-182.

Received June, 2001. Revised January, 2002.

To access this journal online: www.edpsciences.org 